\title{
Hematologist Intervention in Disseminated Intravascular Coagulation - Has Something Changed in 2018?
}

\author{
David Gómez-Almaguer, Emmanuel Bugarin-Estrada and José Miguel Yáñez-Reyes \\ Hematology Service, School of Medicine, "Dr. José Eleuterio González" University Hospital, Autonomous University of Nuevo Leon, NL, Mexico
}

\begin{abstract}
Disseminated intravascular coagulation (DIC) is a systemic condition secondary to an underlying process that affects hemostasis, causing thrombocytopenia and coagulation factor consumption. Severe sepsis, polytraumatic lesions, obstetric complications, cancer, and acute promyelocytic leukemia are, some of the pathologies that can trigger this hemostatic disorder. Despite the severity of DIC, there have been minimal changes in the therapeutic approach over time. The cornerstone of treatment is to identify the etiology and eliminate the underlying cause. On the other hand, it is necessary to replace the lack of blood elements such as platelets and coagulation factors, using fresh frozen plasma or clotting factor concentrates. In this paper, we have reviewed the timeline over 30 years regarding the evolution of therapeutic hematologic support for DIC.
\end{abstract}

KEY WORDS: Coagulation. Treatment. Disseminated intravascular coagulation. Hemorrhage. Plasma. Platelets.

\section{Introduction}

In everyday practice, hematologists, internists or intensive care physicians face relatively often the combination of thrombocytopenia, prolonged prothrombin, and thromboplastin times in the context of a patient with hemorrhage. If we rule out acute or chronic liver disease, which is crucial, this combination of laboratory alterations forces us to think in disseminated intravascular coagulation (DIC). This is often a complication secondary to a severe problem which compromises patient's life. As previously indicated, the patient usually presents some type of acute or chronic but worsening basal disease such as sepsis and some type of cancer, i.e., promyelocytic leukemia, severe trauma, obstetric complications, and, in some cases, intravascular hemolysis. DIC can then be defined as an "acquired syndrome, characterized by the intravascular activation of the coagulation system in a generalized way and which may have different causes." ${ }^{\prime 1}$ The definition by itself is not specific and denotes the lack of an in-depth knowledge on this catastrophic phenomenon.

$\mathrm{DIC}$ is classically described as an abnormal response of hemostasis to an underlying condition. In physiological conditions, hemostasis is regulated by multiple regulatory mechanisms which in DIC do not work properly, this combined with the endothelial damage contributes to the massive production of thrombin in a systemic way ${ }^{2}$.

To confirm the diagnosis, ideally, the combination of thrombocytopenia, elevated D-dimer, and hypofibrinogenemia in the presence of prolonged prothrombin and thromboplastin times leads us to be reasonably sure of the diagnosis ${ }^{3,4}$. Some pathologies can be distracting, foremost among them are severe hepatopathy, thrombotic thrombocytopenic purpura, and hemolytic uremic

\section{Correspondence:}

David Gómez-Almaguer

E-mail: dgomezalmaguer@gmail.com
Available online: 18-01-2019

Date of reception: $10-10-2018$

Date of acceptance: $30-10-2018$

DOI: 10.24875/RMU. 18000028
Medicina Universitaria. 2018;20(4):166-171 www.medicinauniversitaria.org

México SA de CV. This is an open access article under the CC BY-NC-ND license (http://creativecommons.org/licenses/by-nc-nd/4.0/). 


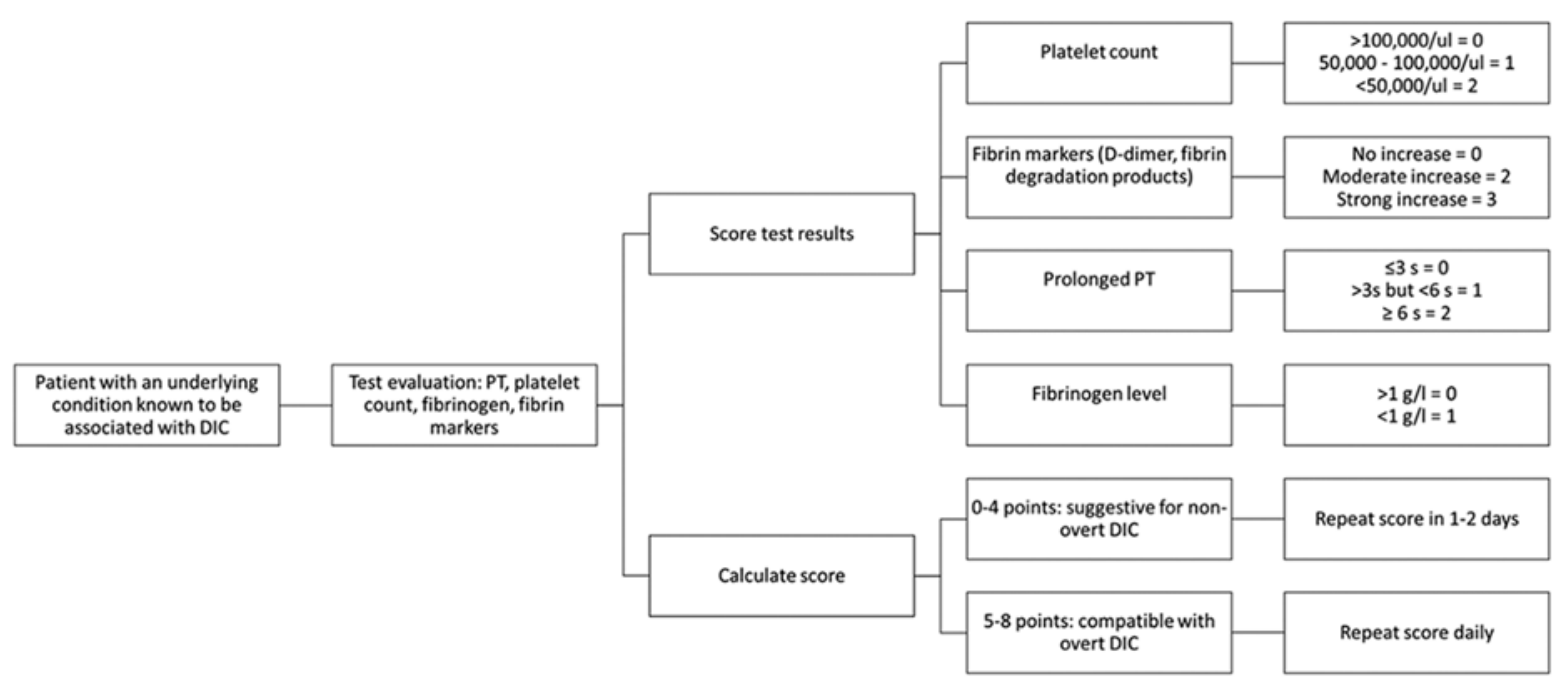

Figure 1. Diagnostic algorithm for patients with suspicion of disseminated intravascular coagulation ${ }^{9}$.

Table 1. Laboratory findings in patients with DIC

\begin{tabular}{|l|l|l|}
\hline $\begin{array}{l}\text { Laboratory } \\
\text { parameter }\end{array}$ & Expected result & Acute versus chronic \\
\hline Platelet count & $\downarrow$ & Acute \\
\hline FDP, D-dimer & $\uparrow$ & Both \\
\hline Fibrinogen & $\downarrow$ & Acute \\
\hline PT & $\uparrow$ & Acute \\
\hline aPTT & $\uparrow$ & Acute \\
\hline
\end{tabular}

DIC: disseminated intravascular coagulation.

syndrome. The presence of thrombosis can cause D-dimer elevation but not other major hemostatic alterations. In table 1, the main laboratory findings are described in patients with DIC, and in figure 1, a practical diagnostic algorithm is described to suspect or rule out this disease.

\section{Analysis and discussion of the therapeutic options of DIC}

In this paper, we decided to evaluate review articles and clinical guidelines found in the MEDLINE database linked to the recommendations for DIC management. From the consulted articles, we chose seven according to the impact of such publications during the past three decades (Table 2) (11. $^{5}$.

The question to answer is quite simple - are there any significant practical changes in the treatment of
DIC on the hematologist perspective? At first, all seven publications concur that treatment is based on support for the patient and solving the triggering cause as it may be treating an infectious process, using a target treatment for promyelocytic leukemia or solve an obstetric complication.

The first article evaluated data from $1987^{5}$ where the therapy used for DIC was based on the application of fresh frozen plasma, cryoprecipitates, and platelet transfusion. In 2018, according to the article published in the section "How I treat" from the journal Blood", the treatment is still based on in these principles; however, it makes a reference to the utility of purified clotting factor concentrates in patients with a high risk of bleeding or with active bleeding.

Evaluating the main antihemorrhagic options for DIC management, Carr Jr. ${ }^{5}$ mentions in his article of 1987 that replacement therapies were previously contraindicated in acute DIC processes. Nevertheless, the use of fresh plasma became accepted as the first line in case of bleeding, and it remains to be recommended today ${ }^{11}$. In 2005, Levi ${ }^{8}$ mentioned that the use of fresh plasma should not be based solely on laboratory studies but to make the decision taking into consideration the clinical state of the patient.

Regarding cryoprecipitates, Car $\mathrm{Jr}^{5}$ stresses in the late 80 s that its use ought to be reserved for patients with severe hypofibrinogenemia $(<50 \mathrm{mg} / \mathrm{dL}$ ) or in cases of active bleeding with fibrinogen levels lower than $100 \mathrm{mg} / \mathrm{dL}$. In 2009, the British Society of Hematology guidelines mentioned that the use of cryoprecipitates 

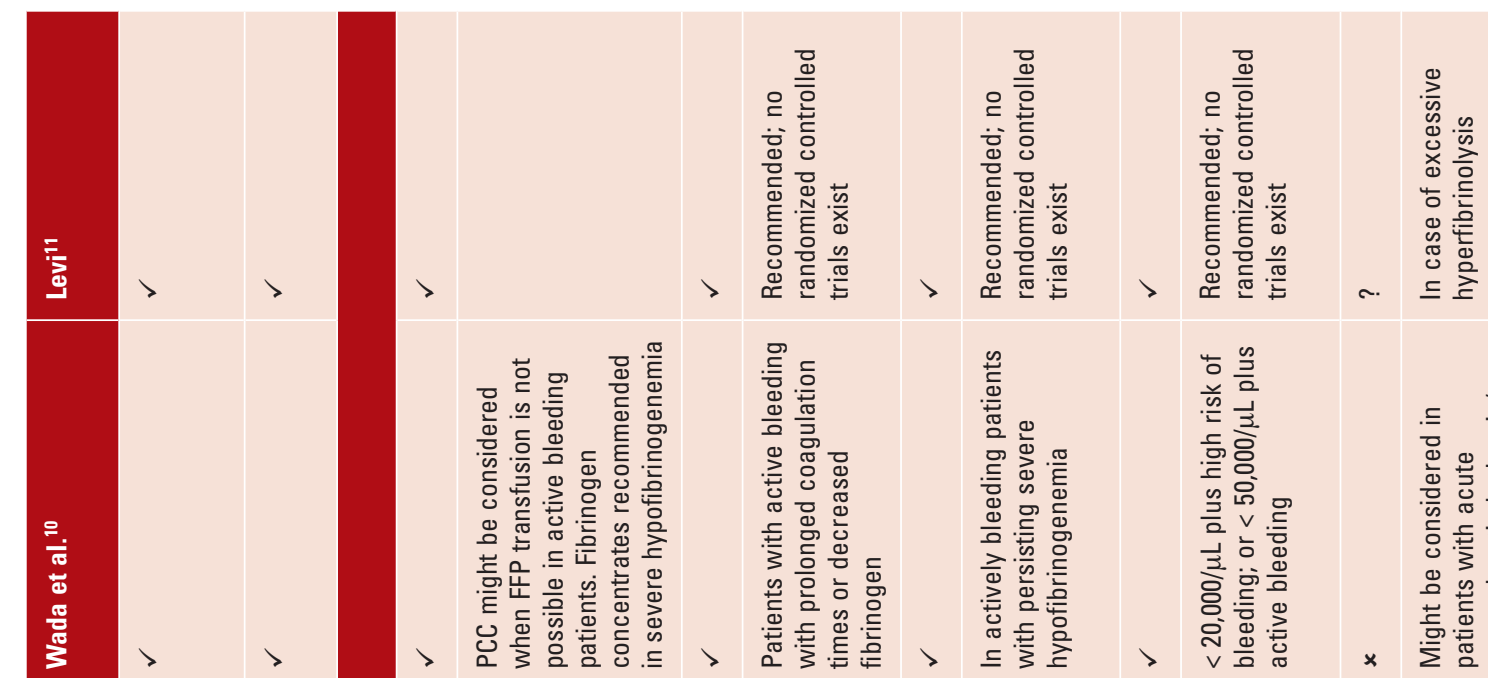

ฮิ

U.
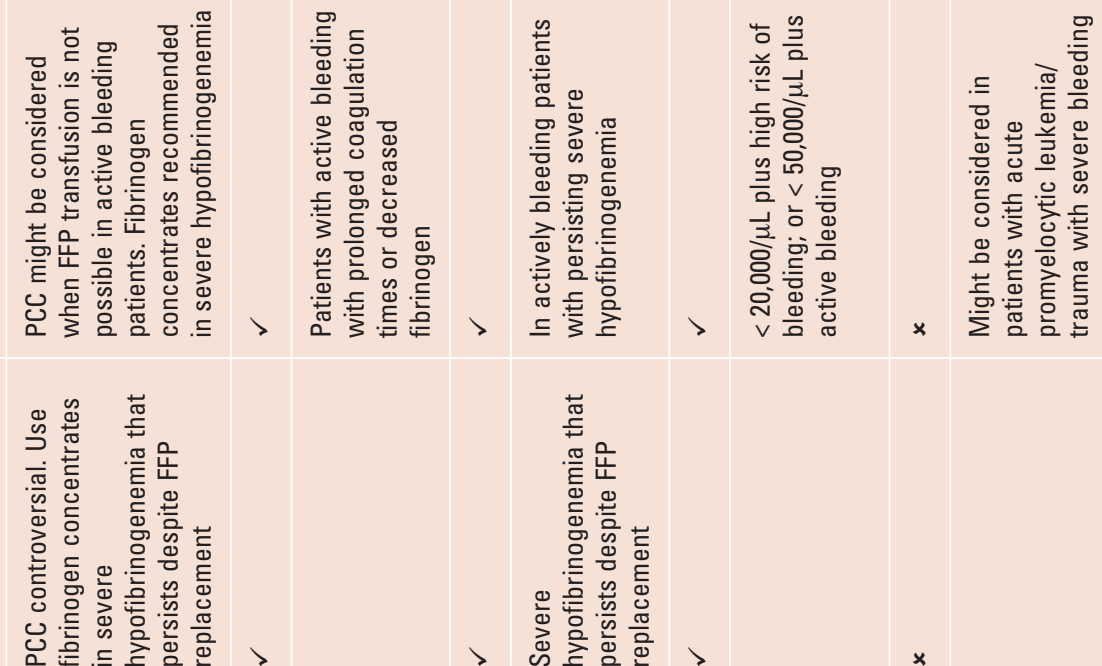

$\stackrel{+\pi}{\leftrightarrows}$
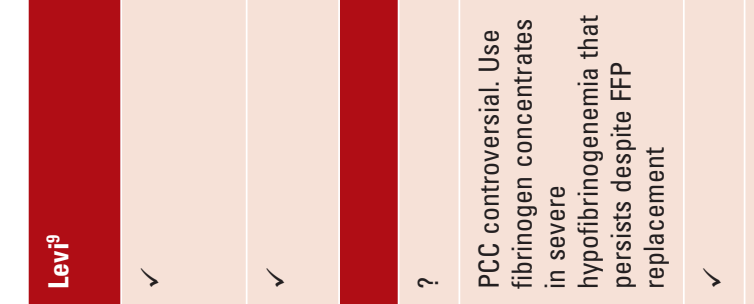

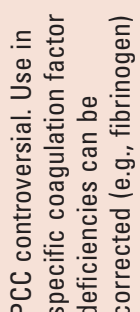

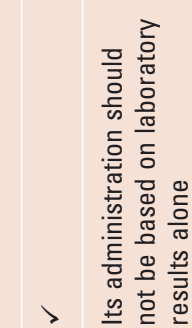

का

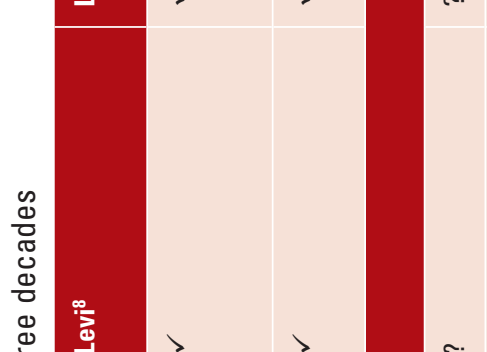

का

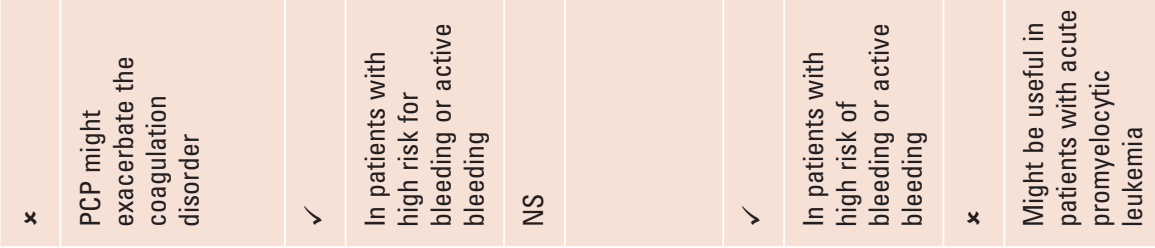

늠

西

.

宽

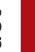

$\stackrel{\oplus}{\rightleftharpoons}$

올

产

言
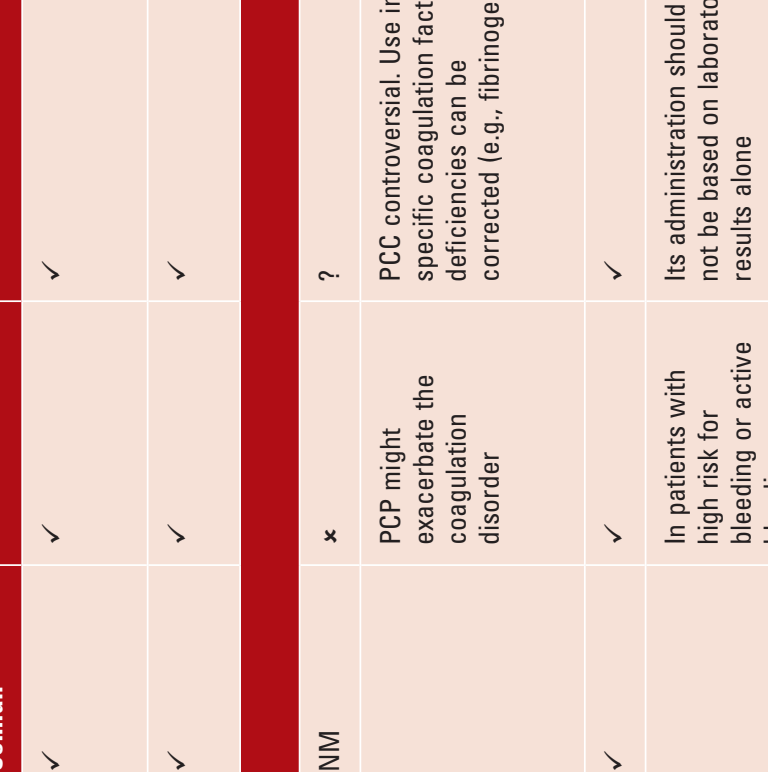

응

客宫: 응

(당

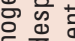

辛

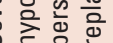

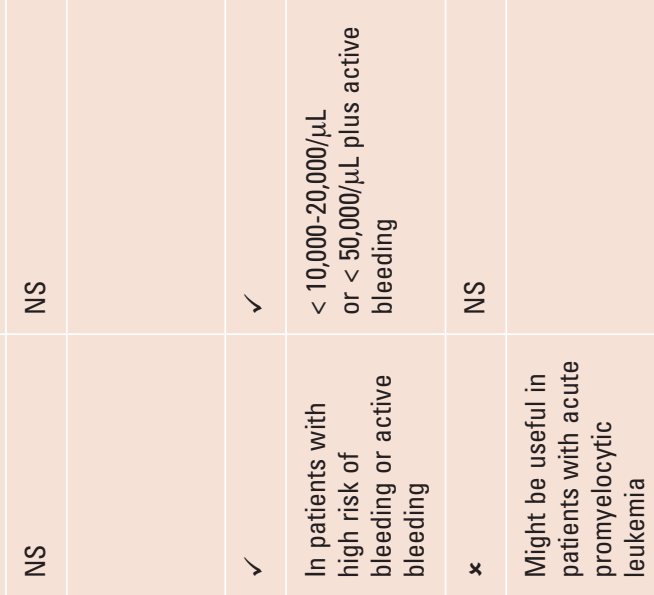

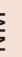

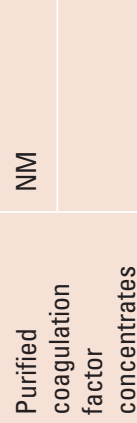

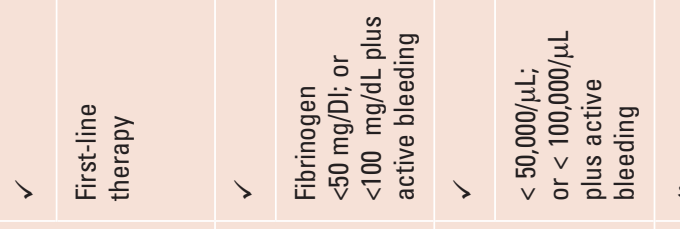
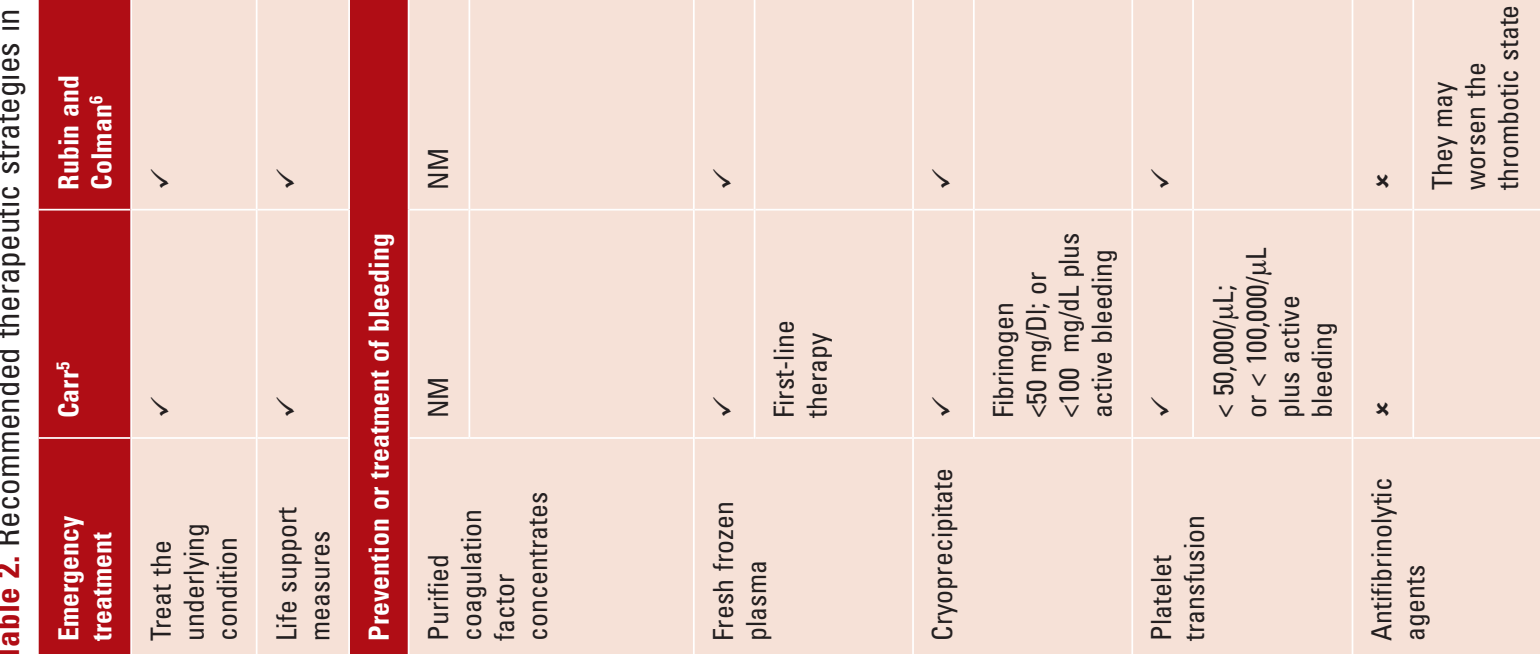

造

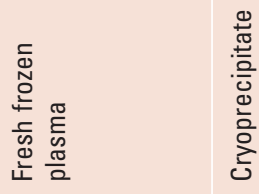

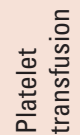

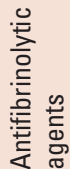




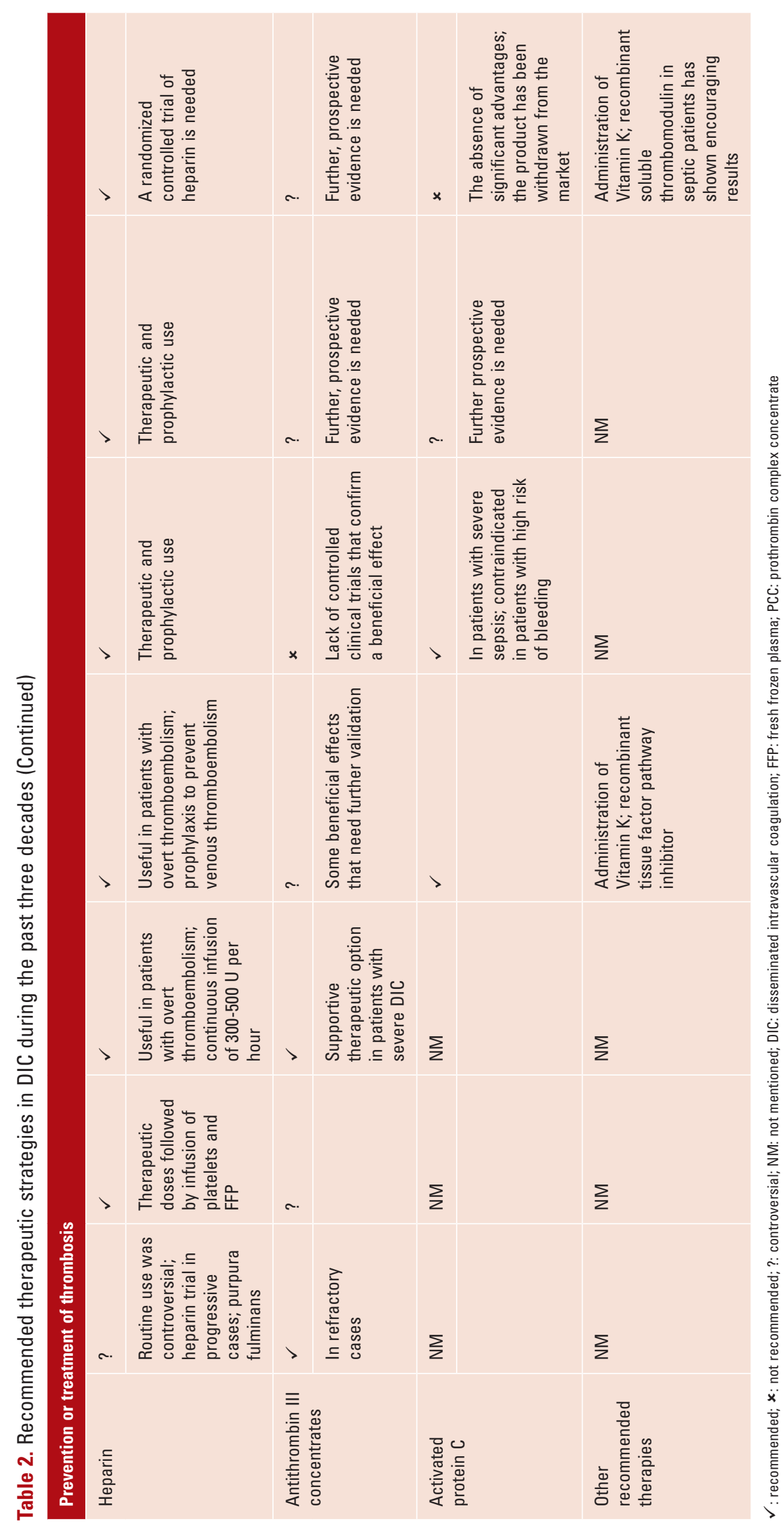


or fibrinogen concentrates ought to be used when there is a persisting hypofibrinogenemia lower than $100 \mathrm{mg} / \mathrm{dL}$ despite fresh frozen plasma replacement ${ }^{9}$. In 2013, its indication was the same but in cases with active bleeding $^{10}$.

Recently, indications for platelet transfusions have decreased cutoff values from $20,000 / \mu \mathrm{L}$ in patients with a risk of bleeding and $50,000 / \mu \mathrm{L}$ with active bleeding ${ }^{8-11}$. Three decades ago, the recommendation for platelet administration in DIC was when having counts lower than $50,000 / \mu \mathrm{L}$ or under $100,000 / \mu \mathrm{L}$ with active bleeding $^{5}$.

When evaluating recommendations about the use of purified clotting factor concentrates, we did not find evidence of its use in two of the oldest articles cited ${ }^{5,6}$. It is up to 1999 that Levi and Ten discussed its application in DIC and did not recommend its application since it could worsen coagulative disorder. In 2005 and 2009, its use remains under controversy since it mentions its possible application in cases with severe hypofibrinogenemia ${ }^{8,9}$. It is until 2013 that Wada et al. ${ }^{10}$ recommend its use in patients with active bleeding where the administration of fresh plasma is not possible or in cases with severe hypofibrinogenemia.

Up to present, there are few randomized clinical studies in adults evaluating or comparing the effectiveness of the standard treatments due to their critical clinical conditions. This is discussed by Squizzato et al. ${ }^{12}$, who tried to find randomized clinical studies which answered to fictional clinical scenarios of DIC, but representative of the most common triggering causes; and at the same time, it is stressed in the article by Levi ${ }^{11}$ published in 2018 , obviously, the resulting evidence of this imaginary essay was poor. In his 2005 article, Levi ${ }^{8}$ mentions that even though the administration of hemocomponents can "add fuel to the fire," the administration of plasma and platelets is clearly necessary in those cases with active bleeding or when there is a high risk of bleeding and/or depletion of these components.

The use of heparin in the context of the DIC has been part of a vicious cycle due to the lack of overwhelming scientific evidence supporting its benefits ${ }^{11}$. However, since 1992, Rubin and Colman ${ }^{6}$ indicated its therapeutic use along with platelets, plasma, and cryoprecipitates when required. As of 2005, its benefits have been considered prophylactically in these patients ${ }^{8}$. On the other hand, it is interesting noting that the use of Vitamin $\mathrm{K}$ continues to be recommended ${ }^{11}$. In addition, thrombomodulin has been utilized with possible benefits due to its interaction with protein $\mathrm{C}^{13}$ yet with low and questionable clinical evidence. It is important to note that the product is not approved for its clinical use in most of the countries around the globe. The use of other products like antithrombin III is yet more objectionable and does not seem to be of any use $e^{14}$. Finally, the lack of commercial availability of activated protein C, in part due to its doubtful usefulness, limits its clinical use ${ }^{15}$.

\section{Conclusion}

Nowadays, DIC represents a severe hemostatic condition; the foundation for its treatment is based on solving the triggering cause and support therapies. For more than 30 years, clotting factor replacement with plasma and platelets has remained as the first-line therapy in the management of bleeding. Recently, the use of other alternatives has been proposed, alternatives with high costs and doubtful benefits like the administration of recombinant clotting factors. It is reasonable to accept that critical conditions in patients to evaluate new treatments difficult, being complicated to carry out all the clinical studies with enough evidence which support their benefits. Unfortunately, there does not seem to be anything new in the treatment of DIC, at least something significant or practical, that is, based on the reports by the experts in the past 30 years.

\section{Conflicts of Interest}

The authors declare no conflicts of interest regarding the publication of this article.

\section{Funding}

None.

\section{Ethical disclosures}

Protection of human and animal subjects. The authors declare that no experiments were performed on humans or animals for this study.

Confidentiality of data. The authors declare that they have followed the protocols of their work center on the publication of patient data.

Right to privacy and informed consent. The authors have obtained the written informed consent of the patients or subjects mentioned in the article. The corresponding author is in possession of this document. 


\section{References}

1. Taylor FB Jr. Toh CH, Hoots WK, et al. Towards definition, clinical and laboratory criteria, and a scoring system for disseminated intravascular coagulation. Thromb Haemost. 2001;86:1327-30.

2. Thachil J. Disseminated intravascular coagulation-new pathophysiological concepts and impact on management. Expert Rev Hematol. 2016;9:803-14

3. Levi M. Pathogenesis and diagnosis of disseminated intravascular coagulation. Int J Lab Hematol. 2018;40 Suppl 1:15-20.

4. Wada H, Matsumo T, Yamashita Y. Diagnosis and treatment of disseminated intravascular coagulation (DIC) according to four DIC guidelines. J Intensive Care. 2014;2:15.

5. Carr ME Jr. Disseminated intravascular coagulation: pathogenesis, diagnosis, and therapy. J Emerg Med. 1987;5:311-22.

6. Rubin RN, Colman RW. Disseminated intravascular coagulation. Approach to treatment. Drugs. 1992;44:963-71.

7. Levi M, Ten Cate H. Disseminated intravascular coagulation. N Engl J Med. 1999;341:586-92.

8. Levi M. Disseminated intravascular coagulation: what's new? Crit Care Clin. 2005;21:449-67.
9. Levi M, Toh $\mathrm{CH}$, Thachil J, Watson HG. Guidelines for the diagnosis and management of disseminated intravascular coagulation. British committee for standards in haematology. Br J Haematol. 2009;145:24-33.

10. Wada H, Thachil J, Di Nisio M, et al. Guidance for diagnosis and treatment of DIC from harmonization of the recommendations from three guidelines. J Thromb Haemost. 2013. Doi: 10.1111/jth.12155.

11. Levi M, Scully M. How i treat disseminated intravascular coagulation. Blood. 2018;131:845-54.

12. Squizzato A, Hunt BJ, Kinasewitz GT, et al. Supportive management strategies for disseminated intravascular coagulation. An international consensus. Thromb Haemost. 2016;115:896-904.

13. Yamakawa K, Aihara M, Ogura $H$, et al. Recombinant human soluble thrombomodulin in severe sepsis: a systematic review and meta-analysis. J Thromb Haemost. 2015;13:508-19.

14. Hayakawa M, Kudo D, Saito S, et al. Antithrombin supplementation and mortality in sepsis-induced disseminated intravascular coagulation: a multicenter retrospective observational study. Shock. 2016;46:623-31.

15. Bernard GR, Vincent JL, Laterre PF, et al. Efficacy and safety of recombinant human activated protein $\mathrm{C}$ for severe sepsis. $\mathrm{N}$ Engl J Med. 2001;344:699-709 